\title{
The MAGIC Study and the Gastrointestinal Effects of Low-Dose Aspirin
}

\author{
Editorial to: "Prospective Cohort Study of Gastrointestinal Complications and Vascular \\ Diseases in Patients Taking Aspirin: Rationale and Design of the MAGIC Study" \\ by $H$. Origasa et al.
}

John McNeil • Andrew Tonkin

Published online: 18 October 2011

(C) Springer Science+Business Media, LLC 2011

In this edition of Cardiovascular Drugs and Therapy, Origasa and colleagues describe the rationale and design of the MAGIC Study, a large Japanese cohort study designed to assess the frequency of gastrointestinal complications associated with low-dose aspirin therapy [1]. Bleeding, particularly from the gastrointestinal tract is the most important adverse effect of aspirin but its frequency and consequences have not been well studied. In most large scale randomised trials the focus has been on the potential benefits with less rigorous assessment of the negative consequences of therapy.

The MAGIC study involves an intensive one year follow-up of 1533 patients with a variety of cardiovascular conditions recruited from throughout Japan. They will have been taking low-dose aspirin in a dose of $81-325 \mathrm{mg} /$ day for at least one month prior to entry. Each patient will undergo endoscopy at entry and after 12 months treatment and any occurrence of significant gastrointestinal bleeding will be investigated.

The study will improve understanding of the effect of chronic low-dose aspirin therapy on gastric mucosa. The study will also be able to relate gastric mucosal injury to dose and measure the frequency of clinical evident bleeding over 12 months. In previous studies where bleeding has been carefully documented its incidence has been surprisingly high [2-5] and it will be interesting to establish if this study confirms these observations.

J. McNeil $(\bowtie) \cdot$ A. Tonkin

Department of Epidemiology \& Preventive Medicine,

School of Public Health and Preventive Medicine,

Monash University,

Alfred Centre, 99 Commercial Road,

Melbourne, Victoria 3004, Australia

e-mail: john.moneil@monash.edu
The investigators are also planning to assess any differences between a variety of subgroups based on such factors as the presence or absence of $\mathrm{H}$. pylori infection or co-prescription of anti-secretory drugs (e.g. proton pump inhibitors). Whether or not these agents should be routinely prescribed with low-dose aspirin therapy (or restricted to those with a past history of gastrointestinal pathology) is debatable. Demonstration that these agents effectively reduced the adverse effects of aspirin on gastric mucosa would inform this debate.

However, there are potential limitations to the MAGIC study which are also inherent in its design. Firstly all of the patients will have had at least one month of prior aspirin therapy. Consequently the cohort will be made up of 'survivors' on aspirin therapy and therefore may underestimate the true risk. The lack of a control group taking either placebo or an alternative anti-platelet drug will also limit the conclusions that can be drawn, as will the modest number of subjects included which may impact on the reliability of the subgroup analyses

Most importantly however, the MAGIC study will provide only limited insights into the clinical significance of these adverse gastrointestinal effects, particularly the extent to which these may negate the benefits of aspirin. This is a question of key importance to clinicians and one which has not been adequately addressed by the reported trials which have mainly focussed on quantifying effects on myocardial infarction and stroke. In patients in whom aspirin is used for secondary prevention the underlying risk is sufficiently high that the benefits are likely to outweigh risks in most clinical settings. However in primary prevention where the risk benefit balance is more finely balanced this question is of greater importance [6-10]. 
In the elderly the trade-off between risks and benefits is probably even more important because gastrointestinal bleeding in patients receiving aspirin increases sharply with age [11]. It is likely that overt gastrointestinal bleeding is only one end of a spectrum and in others bleeding may be slow or intermittent (and subclinical) leading to chronic anaemia and impaired quality of life.

An important focus of current clinical research is the development of 'composite' measures which can inform clinicians whether there is net benefit or net harm from prescribing long term preventive therapy. The US/Australian ASPREE trial of low-dose aspirin versus placebo is one such study [12]. This study of 19,000 apparently healthy subjects aged 70 years and over has the single primary endpoint of 'disability-free survival' which may be influenced positively by a reduction in heart disease, stroke or cancer but negatively impacted by bleeding, anaemia or other adverse effects. ASPREE will clarify the impact of bleeding in the context of the benefits of aspirin and provide clear guidance to physicians about where the balance of risks and benefits lies in the elderly.

In summary the inclusion of endoscopy in the MAGIC study will provide useful information on the effects of chronic low-dose aspirin therapy on the gastric mucosa. This information will add to that provided by other ongoing studies to clarify how much of the benefit of this therapy is offset by adverse effects resulting from gastrointestinal irritation.

\section{References}

1. Origasa H, Goto S, Shimada K, Uchiyama S, Okada Y, Sugano K, et al. Prospective cohort study of gastrointestinal complications and vascular diseases in patients taking aspirin: rationale and design of the MAGIC Study. Cardiovasc Drugs Ther. 2011;25: this issue.
2. Silagy CA, McNeil JJ, Donnan GA, Tonkin AM, Worsam B, Campion K. Adverse effects of low-dose aspirin in a healthy elderly population. Clin Pharmacol Therap. 1993;54:84-9.

3. Slattery J, Warlow CP, Shorrock CJ, Langman MJ. Risks of gastrointestinal bleeding during secondary prevention of vascular events with aspirin - analysis of gastrointestinal bleeding during the UK-TIA trial. Gut. 1995;37:509-11.

4. Lanas A, Wu P, Medin J, Mills EJ. Low doses of acetylsalicylic acid increase risk of gastrointestinal bleeding in a meta-analysis. Clin Gastroenterol Hepatol. 2011;9:762-768.e6.

5. Fujisawa $\mathrm{N}$, Inamori $\mathrm{M}$, Endo $\mathrm{H}$, Uchiyama $\mathrm{T}$, Hosono $\mathrm{K}$, Akiyama $\mathrm{T}$, et al. Incidence of and risk factors for upper gastrointestinal complications in patients taking low-dose aspirin in Japan. Hepatogastroenterol. 2011;58:229-34.

6. Berger JS, Roncaglioni MC, Avanzini F, Pangrazzi I, Tognoni G, Brown DL. Aspirin for the primary prevention of cardiovascular events in women and men: a sex-specific meta-analysis of randomized controlled trials. JAMA. 2006;295:306-13.

7. Raju N, Sobieraj-Teague M, Hirsh J, O’Donnell M, Eikelboom J. Effect of aspirin on mortality in the primary prevention of cardiovascular disease. Am J Med. 2011;124:621-9.

8. Berger JS, Lala A, Krantz MJ, Baker GS, Hiatt WR. Aspirin for the prevention of cardiovascular events in patients without clinical cardiovascular disease: a meta-analysis of randomized trials. Am Heart J. 2011;162:115-124 e2.

9. Huang ES, Strate LL, Ho WW, Lee SS, Chan AT. Long-term use of aspirin and the risk of gastrointestinal bleeding. Am J Med. 2011;124:426-33.

10. Antithrombotic Trialists' (ATT) Collaboration, Baigent C, Blackwell L, Collins R, Emberson J, Godwin J, et al. Aspirin in the primary and secondary prevention of vascular disease: collaborative meta-analysis of individual participant data from randomised trials. Lancet. 2009;373:1849-60.

11. Patrono C, García Rodríguez LA, Landolfi R, Baigent C. Lowdose aspirin for the prevention of atherothrombosis. N Engl $\mathrm{J}$ Med. 2005;353:2373-83.

12. Nelson MR, Reid CM, Ames DA, Beilin LJ, Donnan GA, Gibbs $\mathrm{P}$, et al. Feasibility of conducting a primary prevention trial of low-dose aspirin for major adverse cardiovascular events in older people in Australia: results from the ASPirin in Reducing Events in the Elderly (ASPREE) pilot study. Med J Aust. 2008;189:105-9. 